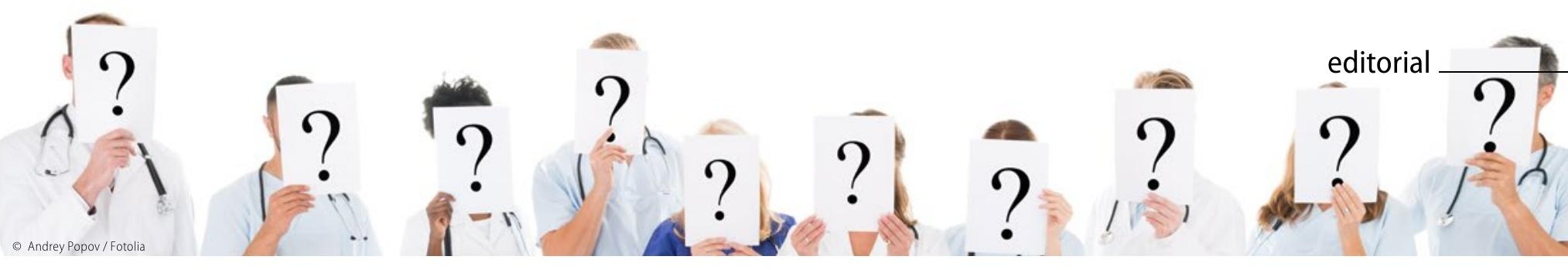

Gut strukturierte Nachsorge verhilft Krebspatienten zu mehr Lebensqualität

\title{
Wer ist verantwortlich für die Krebsnachsorge?
}

Viele Krebspatienten fühlen sich gerade in der Nachsorge orientierungslos, allein gelassen und in hohem Maße verunsichert. Das weiß ich aus meiner Arbeit als Onkologe und als langjähriger Präsident der Bayerischen Krebsgesellschaft e. V. Die Fragen von Patienten, insbesondere zum Thema Nachsorge, bestimmen einen Großteil der Anfragen in den psychosozialen Krebsberatungsstellen der Bayerischen Krebsgesellschaft e.V.

Wünschenswert wäre es, wenn Krebspatienten bereits bei Abschluss der stationären Erstbehandlung gut informiert in die Nachsorge gehen könnten und wüssten, wer ihr verantwortlicher Nachsorgearzt ist. Bei einem solchen Entlassungsgespräch sollte auch festgelegt werden, wann welche Nachsorgeuntersuchungen anstehen. Nur so lässt sich der Behandlungserfolg langfristig sichern und die Lebensqualität der Betroffenen erhalten.

Die Realität sieht allerdings oft anders aus. Während Patienten in der Akutphase ihrer Behandlung nahezu lückenlos betreut werden und auch feste Ansprechpartner haben, werden viele Patienten nach Hause entlassen, ohne ausreichend über die verschiedenen Optionen in der Nachsorge aufgeklärt worden zu sein. Dann beginnt für die Betroffenen eine Zeit der Unsicherheit und der Fragen. Und häufig sind sie aufgrund ihrer körperlichen und seelischen Verfassung nicht in der Lage, Abläufe zu verstehen, nötige Abstimmungen einzufordern und den Überblick zu behalten.

\section{Krebsnachsorge ist Vorsorge}

Die Nachsorge beginnt in der Regel nach Abschluss der akutstationären Behandlung bzw. der Rehabilitation und beinhaltet in der Folge regelmäßige Kontrolluntersuchungen, um eine wiederkehrende oder neu auftretende Krebserkrankung möglichst früh $\mathrm{zu}$ erkennen und zu behandeln. Um die Lebensqualität der Betroffenen zu erhalten, werden in der Krebsnachsorge Beschwerden, Folgeerkrankungen sowie Nebenwirkungen und etwaige Spätfolgen der Tumortherapie erkannt und therapiert. Haben Patienten psychische, seelische oder soziale Probleme, zieht der betreuende Nachsorgearzt einen Psychoonkologen hinzu bzw. verweist an die nächstgelegene Krebsberatungsstelle in der Region. Werden alle Aspekte in der Nachsorge gleichermaßen berücksichtigt, kann eine gut strukturierte und mit allen Akteuren abgestimmte Nachsorge den Behandlungserfolg sichern und die Chancen für ein längeres Überleben mit guter Lebensqualität erhöhen.

\section{Wer hält die Fäden in der Hand?}

Im Rahmen der verschiedenen S3-Leitlienien zur Behandlung häufiger Krebserkrankungen gibt es Nachsorgeempfehlungen. Sie dienen als Orientierungshilfe und lassen Raum für die eige-

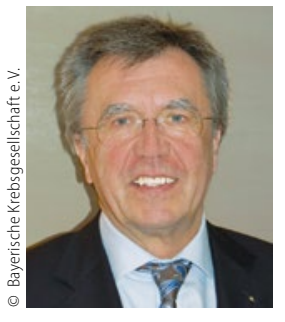

Prof. Dr. Günter Schlimok

Bayerische Krebsgesellschaft e. V.

info@bayerische-krebsgesellschaft.de

ne fachliche Entscheidung. Für alle Patienten allgemeingültige Vorgaben zur Krebsnachsorge gibt es kaum. Je nach Art und Verlauf der Krebserkrankung benötigt jeder Patient eine individuelle Nachsorge. Viele Patienten führen die Nachsorge in einer onkologischen Tagesklinik durch, andere bei onkologisch geschulten Fachärzten in Praxen. Patienten in ländlichen Gegenden gehen häufig zum Hausarzt, weil sie nicht in der Lage sind, weite Fahrten auf sich zu nehmen. Und genau in dieser Individualität liegt auch die große Herausforderung für Patienten und Ärzte, denn sie setzt eine strukturierte Abstimmung und einen gut informierten Patienten voraus.

Die Krebsnachsorge gehört meines Erachtens fest in die Hand von Onkologen und onkologisch tätigen Fachärzten. Sie informieren sich nicht nur regelmäßig über neue Krebstherapien und Medikamente, sondern ziehen auch aktuelle wissenschaftliche Studienergebnisse für die Behandlung und die Nachsorge ihrer Patienten heran. Natürlich sind auch Hausärzte Ansprechpartner für Krebspatienten. Sie sind eine wichtige Stütze in der sprechenden Medizin und helfen entscheidend bei der häuslichen palliativmedizinischen Versorgung von Krebspatienten.

Unterstützende Netzwerke wie das der Deutschen Krebsgesellschaft e. V. und ihrer Landesverbände (www.krebsgesellschaft.de) sind für Ärzte und Patienten in der Nachsorge wichtige Begleiter. In den entsprechenden Beratungsstellen unterstützen geschulte Psychoonkologen Ärzte bei der weiteren psychoonkologischen Betreuung von Krebspatienten. Sie beraten Betroffene und Angehörige, helfen ihnen dabei, neue Perspektiven für ein Leben mit Krebs zu entwickeln und bieten eine ganze Reihe an unterstützenden und stärkenden Maßnahmen an - dazu gehören auch Ernährungsberatung, Sportprogramme oder Entspannungskurse.

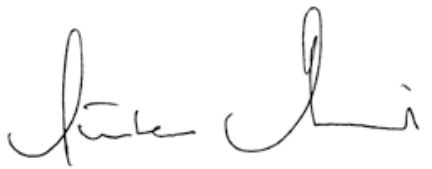

